\title{
Non-thermal high-energy emission from Galactic Wolf-Rayet stars?
}

\author{
Ulf Wessolowski \\ Max-Planck-Institut für Extraterrestrische Physik, \\ $D-85740$ Garching, BRD
}

\begin{abstract}
We have updated the list of promising candidates for observations of non-thermal high-energy emission from Galactic Wolf-Rayet stars with present and future $\mathrm{X} / \gamma$-ray instruments.
\end{abstract}

\section{Introduction}

Theory predicts non-thermal X-ray and $\gamma$-ray continuum emission from winds of luminous massive stars (singles as well as binaries) through at least one of the following three major radiation mechanisms: inverse-Compton scattering of the stellar UV photons by relativistic electrons (Chen \& White 1991a,b); Bremsstrahlung radiation from collisions between electrons and thermal ions (e.g., Pollock 1987a); and $\pi^{\circ}$-decay with the neutral pions produced as an important secondary in ion-ion interactions (White \& Chen 1992). Evidence for the existence of such (pre-requisite) high-energy particle populations stems from the observation of a strong and often variable non-thermal radio component in a number of Wolf-Rayet stars (e.g., Chapman et al. 1999) which is thought to be synchrotron radiation of relativistic electrons accelerated via the first-order Fermi mechanism in strong shocks (White 1985). However, building high-energy source models on stellar wind particle acceleration remains rather speculative before a clear observational proof is found also at $\mathrm{X} / \gamma$-ray energies!

\section{Promising non-thermal candidates sources}

It follows from the above and work on dust formation in the environment of WC stars (e.g., Usov 1991; Williams in these Proceedings) that (necessary?) criteria to be fulfilled by promising candidates for the detection of non-thermal emission at high energy are: (variable) non-thermal radio emission (NT); excess X-ray flux (eX); and episodic/periodic dust formation (IR) (Table 1), as pointed out earlier by van der Hucht (1992). All these phenomena are best met within the model of colliding winds in a long-period binary system for which WR 140 (HD 193793) is the prototype among the WR stars (Williams et al. 1990). Note: At the time of this symposium there seems to be growing evidence for nonthermal X-ray emission of WR 140 in its pre-periastron phase from semi-annual $A S C A$ observations (Corcoran et al. these Proceedings), as preluded already by EXOSAT observations (Williams et al. 1990).

Acknowledgments. The author gratefully acknowledges financial support from the Deutsche Forschungs Gemeinschaft (DFG travel grant We 1388/2-1). 
Table 1. List of (the most?) promising non-thermal candidates sources

\begin{tabular}{rlrrcl}
\hline WR & spectral type & \multicolumn{2}{c}{ gal. coordinates } & criterion & reference(s) \\
& & $\left.l^{\circ}\right)$ & b $\left(^{\circ}\right)$ & & \\
\hline 6 & WN4b (SB1) & 234.76 & -10.08 & (variable X) & WS96,SIN98 \\
11 & WC8+O8.5III & 262.80 & -7.69 & NT: / (var. X) & CLK99/WSS95,SCW96 \\
14 & WC7 & 267.55 & -1.64 & NT & CLK99 \\
19 & WC5+O9.5-7 & 283.89 & -1.19 & IR & W97,VHW98 \\
22 & WN7ha+O7.5 & 287.17 & -0.85 & eX & P87b \\
25 & WN6ha+O4? & 287.51 & -0.71 & eX & P87b \\
39 & WC7 & 290.63 & -0.90 & NT & CLK99 \\
48 & WC6+O9.5I & 304.67 & -2.49 & NT & CLK99 \\
$48 \mathrm{a}$ & WC8 & 305.37 & +0.07 & IR & W97 \\
70 & WC9+B0I & 322.34 & -1.81 & IR & W97 \\
90 & WC7 & 343.16 & -4.76 & NT & CLK99 \\
97 & WN5b+O7 & 354.68 & -1.12 & eX & P87b \\
98 & WN80/WC7 & 355.21 & -0.87 & NT: & ABCT86 \\
$98 \mathrm{a}$ & WC9 & 358.13 & -0.03 & IR & W97 \\
103 & WC9 (SB1?) & 358.48 & -4.89 & ('dusty' WC) & VGH98 \\
104 & WC9+B0.5V & 6.44 & -0.49 & ('dusty'WC) & C97 \\
105 & WN9h & 6.52 & -0.52 & NT & CLK99 \\
112 & WC9 & 12.15 & -1.19 & NT & CLK99 \\
113 & WC8+O8-9IV & 18.91 & +1.75 & ('dusty'WC) & VGH98 \\
121 & WC9 & 28.73 & -0.13 & ('dusty'WC) & VGH98 \\
125 & WC7+O9III & 54.44 & +1.06 & NT / eX / IR & CLK99/P87b/W97 \\
137 & WC7+OB & 74.33 & +1.09 & IR & W97 \\
139 & WN5o+O6 & 76.60 & +1.43 & (variable X) & CSP96, MKY99 \\
140 & WC7+O4-5 & 80.93 & +4.18 & NT / eX / IR & P87b/W90/W97/CLK99 \\
144 & WC4 & 80.04 & +0.93 & NT: & ABCT86 \\
145 & WN7o/WCE+OB? & 79.69 & +0.66 & eX & P87b \\
146 & WC5+O8.5V & 80.56 & +0.45 & NT & CLK99 \\
147 & WN8(h)+B0.5V & 79.85 & -0.32 & NT & CLK99 \\
\hline
\end{tabular}

\section{References}

Abbott, D.C., Bieging, J.H., Churchwell, E.B., Torres, A.V. 1986, ApJ 303, 239

Chapman, J.M., Leitherer, C., Koribalski, B. et al. 1999, ApJ in press (CLK99)

Chen, W., White, R.L. 1991a, ApJ 366, 512

Chen, W., White, R.L. 1991b, ApJ 381, L63

Corcoran, M.F., Stevens, I.R., Pollock, A.M.T., et al. 1996, ApJ 464, 434 (CSP96)

Crowther, P.A. 1997, MNRAS 290, L59 (C97)

van der Hucht, K.A. 1992, The A\&A Rev. 4123

Maeda, Y., Koyama, K., Yokogawa, J. 1999, ApJ 510, 967 (MKY99)

Pollock A.M.T., 1987a, A\&A 171, 135

Pollock A.M.T., 1987b, ApJ 320, 283 (P87b)

Skinner S.L., Itoh M., Nagase F., 1998, NewA 3, 37 (SIN98)

Stevens I.R., Corcoran M.F., Willis A.J., et al. 1996, MNRAS 283, 589 (SCW96)

Usov V.V., 1991, MNRAS 252, 49

Veen P.M., van Genderen A.M., van der Hucht K.A., et al. 1998, A\&A 329, 199 (VGH98)

Veen P.M., van der Hucht K.A., Williams P.M., et al. 1998, A\&A 339, L45 (VHW98)

White R.L., 1985, ApJ 289, 698

White R.L., Chen W., 1992, ApJ 387, L81

Williams P.M., van der Hucht, K.A., et al. 1990, MNRAS 243, 662 (W90)

Williams P.M., 1997, ApSS 251, 321 (W97)

Willis A. J., Stevens I.R., 1996, A\&A 310, 577 (WS96)

Willis A. J., Schild H., Stevens I.R., 1995, A\&A 298, 549 (WSS95) 